\title{
RECUPERACIÓN DE LA MEMORIA CULTURAL DEL MUNICIPIO DE PUEBLORRICO, ANTIOQUIA, EN SUS CIEN AÑOS DE VIDA MUNICIPAL
}

\author{
Alirio Valencia Agudelo, ${ }^{68}$ Orlando de Jesús Pineda ${ }^{69}$ y \\ Claudia María Sánchez ${ }^{70}$
}

\begin{abstract}
RESUMEN
El proyecto está encaminado a recuperar la memoria cultural del municipio de Pueblorrico, Antioquia, con motivo de sus cien años de vida municipal, a través de una metodología de acción participación, donde la comunidad se convierte en actor investigado e investigador. Para ello se conformaron grupos de trabajo que abordarán las áreas de historia local, economía y ecología, educación y escuela, religión y parroquia, cultura y deporte, recursos humanos y vida administrativa.
\end{abstract}

Palabras clave: memoria, cultura, historia, desarrollo, comunidad, progreso, educación, organización, convivencia.

\begin{abstract}
The project is directed to recover the cultural memory of Pueblorrico's town, Antioquia, on the occasion of its hundred years of municipal life, across a methodology of action participation, where the community turns into investigated and investigative actor. For it there were conformed workgroups that will approach the areas of local history, economy and ecology, education and school, religion and parish, Culture and sport, human resources and administrative life.
\end{abstract}

Key words: Memory, Culture, History, Development, Community, Progress, Education, Organization, Living Together.

\section{INTRODUCCIÓN}

"Todo pueblo sin memoria y sin identidad está condenado al olvido y a repetir su propia historia. Cuando se carece de sentido de pertenencia a ese terruño donde se vive, cuando no se tiene noción de la historia de ese espacio y de ese entorno, cuando no hay conciencia del

68 Comunicador social de la Universidad Nacional Abierta y a Distancia, UNAD, docente cead Medellín. Correo electrónico: alirio. valencia@unad.edu.co Grupo de investigación: Comunidad, Conflicto y Desarrollo, Código de registro del grupo en Colciencias: COL0094118, Nombre del semillero: SICODES, Línea de investigación: Desarrollo humano, convivencia y productividad, Red de investigación: Desarrollo comunitario. UNAD.

69 Escuela de Ciencias Sociales, Artes y Humanidades, ECSAH, unAD.

70 Escuela de Ciencias Sociales, Artes y Humanidades, ECSAH, UNAD. 
propio destino y no se tienen testimonios, entonces se habita en la nada, en algo así como un limbo existencial: no hay futuro y se hace parte del vacío", dice Reinaldo Spitaleta.

Es por ello que el presente proyecto está encaminado a contribuir con el desarrollo de una comunidad de 10000 habitantes del suroeste antioqueño, toda vez que la realización del mismo se convierte en un aporte necesario y significativo al conocimiento y rescate de la historia, a la búsqueda de la identidad y, que sin duda, contribuirá a aumentar el sentido de pertenencia de cada uno de sus habitantes con el entorno particular: con la calle, con la iglesia, con la vereda, con la plaza llena de toldos en un mercado dominical... con la vida cotidiana.

Para la Universidad Nacional Abierta y a Distancia, UNAD, es sumamente importante saber que mediante el desarrollo de este proyecto se promueve, no sólo la investigación sino, además, la proyección social, toda vez que se está recuperando la historia y toda ese legado cultural que el pasado ha dejado a una importante comunidad, para que así las generaciones futuras conozcan a fondo de dónde vienen y se comprometan con el devenir de un pueblo que, a pesar de las vicisitudes y olas de violencia, ha sabido sostenerse en pie, firme y con una mirada futurista.

Para el desarrollo de esta propuesta se ha vinculado la alcaldía del Municipio de Pueblorrico, la Gobernación de Antioquia y el Ministerio de Cultura; la Alcaldía, facilitando los archivos, documentación, divulgación y espacios para la realización de conversatorios con la comunidad y socialización de resultados. La Gobernación de Antioquia y el Ministerio de Cultura asignando los recursos para la publicación de un libro que se entregará a la comunidad en el mes de marzo de 2011, con motivo de la celebración del Centenario del Municipio.

\section{OBJETIVO GENERAL}

Recuperar la memoria cultural del Municipio de Pueblorrico, Antioquia, en sus cien años de vida municipal, a través de una metodología de Investigación, Acción, Participación, (IAP) que permita la vinculación de la comunidad en todo el proceso para que así las actuales y futuras generaciones conozcan su rico legado histórico y cultural.

\section{OBJETIVOS ESPECÍFICOS}

Recuperar la historia del Municipio de Pueblorrico, Antioquia, desde el momento de su elevación a la categoría de Municipio a la fecha.

Promover el aumento del sentido de pertenencia de la comunidad por su terruño y por el entorno donde vive.

Capacitar a la comunidad pueblorriqueña en temas como la elaboración de proyectos, participación comunitaria y convivencia pacífica. 
Divulgación de la memoria cultural del municipio a través de la publicación de un libro o enciclopedia, que luego pueda trabajarse desde las aulas de clase en los establecimientos de primaria y bachillerato.

\section{METODOLOGÍA}

El proyecto "Recuperación de la Memoria Cultural del Municipio de Pueblorrico, Antioquia" se realizará a través del método de Investigación, Acción, Participación, donde la comunidad se convierte en protagonista de la investigación al hacer parte del equipo que la adelanta, toda vez que se organizarán, capacitarán y trabajarán por equipos de acuerdo con unas áreas propuestas en el proyecto. Las fases a desarrollar son las siguientes:

- Socialización y divulgación del proyecto: en esta primera fase se convocará a los distintos grupos organizados y comunidad en general del Municipio de Pueblorrico, como docentes, estudiantes, líderes campesinos, empleados públicos, amas de casa, comerciantes, etc., para presentar y socializar el proyecto.

- Organización de equipos por áreas de trabajo: luego de socializar el proyecto con la comunidad, se escogerá a cada una de las personas que lideraran los equipos de trabajo para abordar las siguientes áreas: historia local, educación y cultura, religión y parroquia, economía y medio ambiente, recreación y deportes, recursos humanos, vida política y administrativa. Para ello, el investigador principal se convertirá en el Coordinador y asesor de los distintos equipos.

- Capacitación comunitaria: después de conformado cada uno de los equipos de trabajo, a los integrantes se les brindará la respectiva capacitación en lo referente a técnicas de recolección de la información, procedimiento para la realización de entrevistas y consulta de archivos, sistematización de la información y organización de informes.

- Recolección de la información: en esta etapa, la más larga y dispendiosa, los equipos se multiplicarán y aprovecharán todo el recurso humano de la comunidad para iniciar un trabajo de campo debidamente planeado y organizado donde se tenga un contacto directo con las diferentes instituciones y comunidad del área urbana y rural, apoyado en formatos que permitan una adecuada recolección de la información.

- Sistematización de la información: esta etapa se considera que debe ser permanente durante el desarrollo del proyecto, ya que será liderada por el equipo de investigadores, quienes estarán atentos a recibir la información de parte de los equipos para sistematizarla y así organizar el informe final.

- Socialización de resultados: luego de que se tiene toda la información, debidamente sistematizada, se organizarán unos conversatorios y talleres con la comunidad, a fin de socializar la información y aclarar posibles dudas frente a datos recolectados. 
- Presentación del informe final: esta etapa estará a cargo del equipo de investigación quien después de haber socializado y despejado dudas frente a la información recolectada se dedicarán a organizar el informe final, acorde a normas de buena escritura y presentación de trabajos escritos

- Publicación de la investigación: uno de los objetivos de este proyecto y de la comunidad pueblorriqueña es lograr publicar un texto escrito donde se plasme toda su memoria cultural y que quede para conocimiento de las actuales y futuras generaciones. Para ello ya tenemos el compromiso de la actual Administración Municipal y la Gobernación de Antioquia, quienes se vincularán con la publicación de la obra, la cual se presentará a la comunidad en el mes de marzo de 2011, con motivo de la celebración del Centenario del Municipio de Pueblorrico.

- Tipo de estudio: para la realización del proyecto se acudió a un tipo de estudio de Investigación Exploratoria, toda vez que se efectúa sobre un tema u objeto poco estudiado, por lo que sus resultados constituyen una visión aproximada de dicho objeto, es decir, un nivel superficial de conocimiento, dado que se carece de información suficiente y de conocimiento previos del objeto de estudio. Para ello se cuenta con una población de 9.000 habitantes, de los cuales se tomará una muestra aproximada del $1 \%$, aplicando un diseño analítico o explicativo.

- Método: toda vez que se pretende recuperar la historia de una población y una comunidad, el presente proyecto utilizará un método histórico, el cual está vinculado con conocimiento de las distintas etapas de los objetos en su sucesión cronológica, para conocer la evolución y desarrollo del objeto o fenómeno de investigación y se hace necesario revelar su historia, las etapas principales de su desenvolvimiento y las conexiones históricas fundamentales.

\section{Análisis estadístico}

Resultados parciales: el trabajo se inició en el mes de enero del presente año y se encuentra en la primera fase. Para ello se ha acudido a la consulta de archivos y realización de trabajo de campo para la recolección de información, labor que se hace a través de conversatorios y realización de entrevistas con personas de avanzada edad, acudiendo especialmente a la memoria oral.

Perspectivas del trabajo: Pueblorrico es un municipio del suroeste antioqueño, fundado en 1886 y erigido como municipio en 1911, es decir, está próximo a cumplir cien años de vida municipal. Es un siglo de historia, de múltiples vivencias, de desarrollo cultural, de logros y fracasos que la comunidad desconoce, toda vez que no está registrado en ningún texto. Sólo está en algunos documentos de archivo o en el imaginario de los viejos o personas de la tercera edad que les correspondió vivir parte de aquella historia, y que es necesario rescatar a través de la tradición oral y la consulta de archivos, para que finalmente se plasme en un libro y sea de conocimientos de las presentes y futuras generaciones. 
Discusión: Herodoto de Halicarnaso (480-430 a.de C.) llamado al padre de la historia, al escribir su obra "Historias" conocida con el título de "Los nueve libros de la historia", puso el primer hito para el largo y hermoso camino de la ciencia que investigaría y expondría los "actos específicamente humanos" mediante la pesquisa de testimonios dignos de fe, como lo dice en el proemio: "para que las cosas hechas por los hombres no se olviden con el tiempo". En el largo proceso de la investigación histórica la metodología fue perfeccionándose gracias a las diversas escuelas y autores que las enriquecieron, hasta llegar al método de la historia científica.

Esta propuesta, que constituye el primer esfuerzo meritorio para historiar el nacimiento y formación del núcleo social de Pueblorrico y su ubicación dentro de los municipios antioqueños, se convierte en un esfuerzo necesario para impedir que la memoria cultural quede perdida en el olvido. Es una propuesta donde se encontrarán varios de los elementos de la perceptiva metodológica propios del quehacer historiográfico, tales como: recurrencia a las fuentes prístinas (documentos únicos de archivos) y otros de diferentes acervos; consultas de fuentes bibliográficas; análisis de fenómenos políticos y sociales, entrevistas a personas que fueron testigos por varios años del devenir del pueblo, recurriendo a la fuente de la tradición oral, que, careciendo del rigor del método científico, ha sido revaluada plenamente, a falta de documentos escritos. Así vemos que la fuente oral ha servido para escribir la historia, llenando espacios donde no existe lo escrito. Los aportes de los informadores o relatores serán valorados con amplio beneficio de credibilidad dentro de la subjetividad, pues es necesario analizar la información desde el punto de vista de residuo memorístico, sometido a varias limitantes del informante o entrevistado: edad, fijación cronológica del hecho, claridad y lucidez mental, autoridad intelectual, posible interés personal, etc. Es por ello que en el presente trabajo se tendrán en cuenta los conceptos y teorías de aquellos autores que trazan el camino para saber abordar procesos de recolección de información, acudiendo a la tradición oral.

\section{CONCLUSIONES}

En los actuales momentos el proyecto se desarrolla en su primera etapa y cuenta con la participación de docentes, funcionarios públicos, líderes comunitarios, estudiantes y comerciantes, con quienes se conformó 6 grupos de trabajo para abordar las diferentes áreas, destacándose los siguientes aspectos:

Amplia acogida del proyecto por parte de la comunidad pueblorriqueña y Administración Municipal para el desarrollo de la propuesta.

Capacitación de la comunidad para el desarrollo del trabajo de campo.

Apoyo financiero por parte de la Gobernación de Antioquia y el Ministerio de Cultura para la publicación de los resultados. 


\section{REFERENCIAS BIBLIOGRÁFICAS}

ANUARIO Estadístico de Antioquia, varios años.

ARCHIVOS de la Alcaldía y el Concejo Municipal de Pueblorrico.

ARCHIVOS de la Casa Cural del Municipio de Pueblorrico.

VALENCIA, Alirio. (1999), Pueblorrico un pueblo en busca de su identidad. Imprenta Departamental de Antioquia, Medellín.

ZAPATA, Heriberto. (1972), Monografía de los municipios de Antioquia. Editorial Lealón, Medellín 1972. 